\title{
Cooperación científica internacional en la nanomedicina de México
}

\author{
Eduardo Robles Belmont*
}

\begin{abstract}
En este artículo analizamos algunos indicadores sobre las colaboraciones internacionales científicas en el campo de la nanomedicina de México. Los patrones mostrados en los resultados dan una imagen del rumbo que toma el desarrollo de este campo emergente en el país.
\end{abstract}

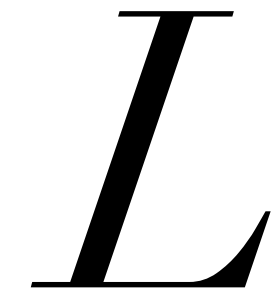

NANOTECNOLOGÍA es una de las últimas tecnologías que ha creado muchas expectativas en torno de sus potencialidades técnicas, económicas y sociales. La biotecnología, la genómica y las tecnologías de la información son otras tecnologías que han creado expectativas importantes. Estas potencialidades se reflejan en muchas áreas de la ciencia, la tecnología e incluso la industria. En los medios de divulgación

\footnotetext{
* Investigador del Laboratorio de Redes del Instituto de Investigaciones en Matemáticas Aplicadas y en Sistemas de la Universidad Nacional Autónoma de México. Miembro de la Red Latinoamericana de Nanotecnología y Sociedad.
}

científica es frecuente encontrar publicaciones sobre las nuevas propiedades de diversos materiales y sus aplicaciones en productos de consumo cotidiano, que de hecho ya son una realidad. El sector salud es uno de los campos donde la nanotecnología está encontrando un nicho importante de aplicación. A esta área se le conoce como nanomedicina y se trata de aplicaciones en diagnósticos, tratamientos, prevención de enfermedades, lesiones traumáticas y medicamentos, entre otras, para la solución de problemas de la salud humana.' Una de las primeras aplicaciones en el sector salud ha sido el desarrollo de microdispositivos electrónicos para el diagnóstico médico (conocidos como Lab-onchip) que actualmente son frecuentes en el mercado y permiten que profesionales de la salud e incluso pa- 
cientes puedan efectuar ciertos diagnósticos sin la necesidad de estar presentes en laboratorios clínicos. El sector de la farmacología se ha inclinado hacia la nanomedicina para impulsar el desarrollo de nuevos medicamentos frente a las limitantes que ha encontrado en los últimos años para innovar. Según los promotores de este campo, estas aplicaciones en la nanomedicina son de suma importancia para el tratamiento y prevención de enfermedades como el cáncer, el VIH y la diabetes, entre otras, que afectan a las sociedades tanto del hemisferio norte como del sur. Sin embargo, las dinámicas que observamos en el desarrollo de la nanomedicina también conciernen a aspectos que requieren regulación, supervisión y monitoreo: la desigualdad en el acceso a los desarrollos nanomédicos y los riesgos a la exposición a nanopartículas y nanomateriales manufacturados. ${ }^{2}$

Estas dinámicas que constatamos al observar el desarrollo de la nanomedicina nos lleva a preguntas sobre las implicaciones sociales de estas nuevas tecnologías, y su comprensión requiere de estudios sociales sobre la producción, el uso y la diseminación de nuevos conocimientos. Una de las cuestiones es la colaboración científica que mantienen los investigadores con sus pares en el extranjero y el perfil de la investigación que desarrollan. En este trabajo presentamos algunos resultados sobre el estudio de la estructura de las colaboraciones científicas internacionales en el campo de la nanomedicina en México. Los resultados nos muestran las tendencias de estas colaboraciones, en las cuales observamos una fuerte concentración con Estados Unidos y países de la Comunidad Europea y poca presencia en la región latinoamericana.

\section{Producción científica en nanotecnologías en MÉxico}

Antes de exponer los resultados sobre las colaboraciones científicas que han mantenido los investigadores mexicanos en el campo de la nanomedicina con el extranjero, es pertinente explicitar algunos puntos sobre la producción de estas tecnologías en el país.

En un trabajo previo se ha caracterizado la evolución del desarrollo de las nanociencias y nanotecnologías en México. ${ }^{3}$ Allí se ha constatado que el desarrollo de estas nuevas tecnologías inició prácticamente en la década de $199^{\circ}$ y que las actividades de investigación se concentran en las principales universidades públi- cas del país. Además, la comparación de los artículos publicados en coautoría con investigadores extranjeros y los artículos publicados sin coautoría con estos últimos ha mostrado que las colaboraciones extranjeras han sido más numerosas que en los trabajos solamente mexicanos hasta 2000. A pesar de que en ese año se observa un cambio en el panorama de las colaboraciones científicas con el extranjero, estas últimas siguen siendo importantes y continúan aumentando. Por otro lado, en aquel primer estudio se identificó que los institutos nacionales de salud ocupan el sexto lugar en la producción en nanociencias y nanotecnologías. De hecho, esto llamó nuestra atención y fue una de las primeras pistas para acercarnos al estudio de la nanomedicina.

El desarrollo de las nanotecnologías ya es una realidad en México, donde existen diversos grupos de investigadores que trabajan en proyectos para el desarrollo de nuevos materiales y moléculas con aplicaciones médicas. Estas actividades se realizan principalmente en laboratorios universitarios y la presencia de hospitales públicos en la investigación es cada vez mayor. El sector privado, ya sea universidades, hospitales o industria farmacéutica, es poco presente en la producción científica. Sin embargo, un panorama diferente podríamos encontrar si revisamos las patentes en el sector de la nanomedicina. Esta última tarea ha quedado pendiente y, a nuestro entender, no hay un estudio de patentes en este campo en México.

Los resultados que presentamos en este estudio están basados en un ejercicio de caracterización de la producción científica en el campo de la nanomedicina en México. Para ello ha sido necesario desarrollar una estrategia de búsqueda de datos adaptada al caso de México. Partimos de la propuesta metodológica de Wagner y colaboradores, ${ }^{4}$ y consultamos la base de datos Web of Science.

Colaboraciones científicas internacionales en la nanomedicina de México

Para identificar las colaboraciones científicas en el campo de la nanomedicina partimos del análisis de coautorías de los artículos identificados y sólo hemos considerado los artículos en coautoría con investigadores extranjeros o localizados fuera de México. Esto nos arrojó un total de zo artículos publicados en colaboración con el extranjero, lo que representa 43.47 por ciento de 


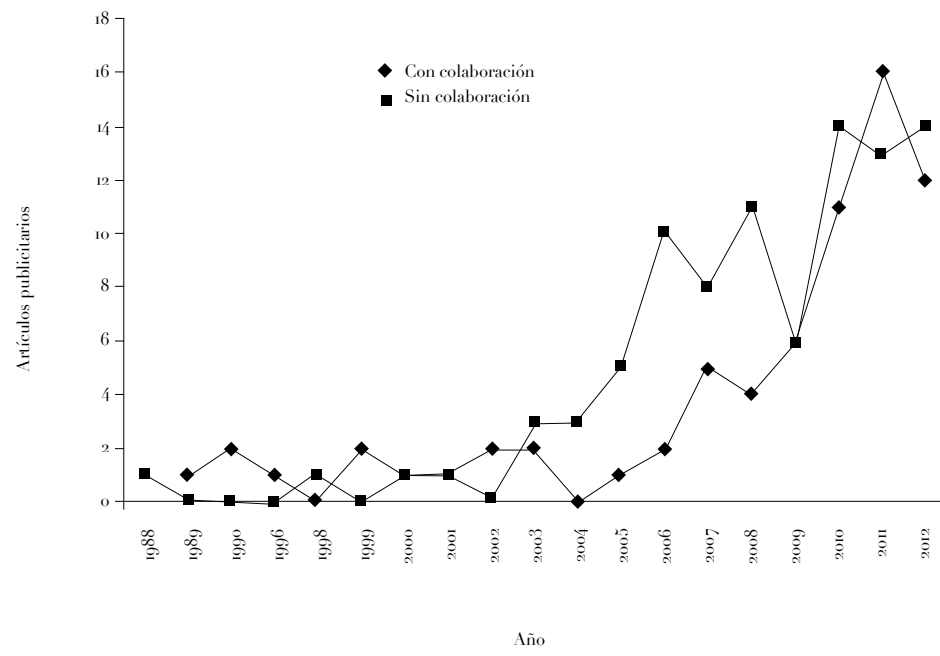

Fuente: Elaboración propia con datos obtenidos de la wos.

los artículos publicados hasta 2012 en este campo. Al comparar la evolución de la producción de artículos publicados en colaboración con el extranjero y aquellos sin colaboración, vemos que ambos tipos de publicaciones se encuentran en estado emergente (véase gráfica i). Además, al observar el crecimiento de ambos tipos de publicaciones, podemos apreciar un crecimiento importante en todo el periodo, principalmente a partir de mediados de la primera década de este siglo.

En diversos estudios sobre el desarrollo de la nanomedicina se ha mencionado que este campo emergente atrae mucho la atención de actores privados por su potencial económico. Surge entonces la pregunta sobre cuál es la presencia de empresas privadas en las colaboraciones científicas que ha mantenido México en el campo de la nanomedicina a nivel internacional. En la base de datos de artículos sobre nanomedicina en este país sólo en dos artículos en colaboración con el extranjero han participado cuatro empresas privadas de origen extranjero. Llama la atención que sobresale la industria militar en las áreas de estas empresas; tres de las cinco empresas encontradas desarrollan actividades en torno de la tecnología bélica. Al revisar la lista de las instituciones mexicanas donde se han producido estos artículos, constatamos que la mayoría son públicas, a excepción del Instituto Tecnológico de Estudios Superiores de Monterrey (ITESM), con dos artículos.

El conteo de las publicaciones en colaboración con el extranjero en este estudio muestra que el principal país colaborador es Estados Unidos, con quien se han publicado en coautoría 33 artículos en nanomedicina; en segundo lugar España, con 15 artículos en coautoría; Cuba en tercer lugar, con nueve artículos, y Francia ocupa la cuarta posición, con ocho artículos. El resto de los países colaboradores han publicado con México menos de cinco artículos.

En este tipo de estudios es interesante visualizar estas colaboraciones, ya que son un reflejo de las redes científicas, así como del alcance y de la posición de México a nivel internacional en el desarrollo de este campo emergente. En la figura i, se muestran las principales redes científicas que han mantenido los investigadores mexicanos con investigadores de otros países. Las líneas representan las colaboraciones a través de las coautorías de artículos y el grosor de las líneas está definido en función de las frecuencias de las coautorías entre los países. Estas redes científicas mostradas sobre un mapa nos aportan una visión muy ilustrativa de la configuración de las colaboraciones científicas en la nanomedicina a nivel internacional. Por ejemplo, en el mapa podemos apreciar que el principal colaborador es Estados Unidos, como ya se mencionó. Además, podemos ver que en las redes científicas con este país participan investigadores de otros países como España, Argentina y Francia, entre otros.

En el mapa se puede apreciar el peso de éstas con Estados Unidos y Europa. Sin embargo, el panorama de las colaboraciones con los países de América Latina y el Caribe presenta pocas relaciones de colaboración, a pesar de que con Cuba se han mantenido colabora- 


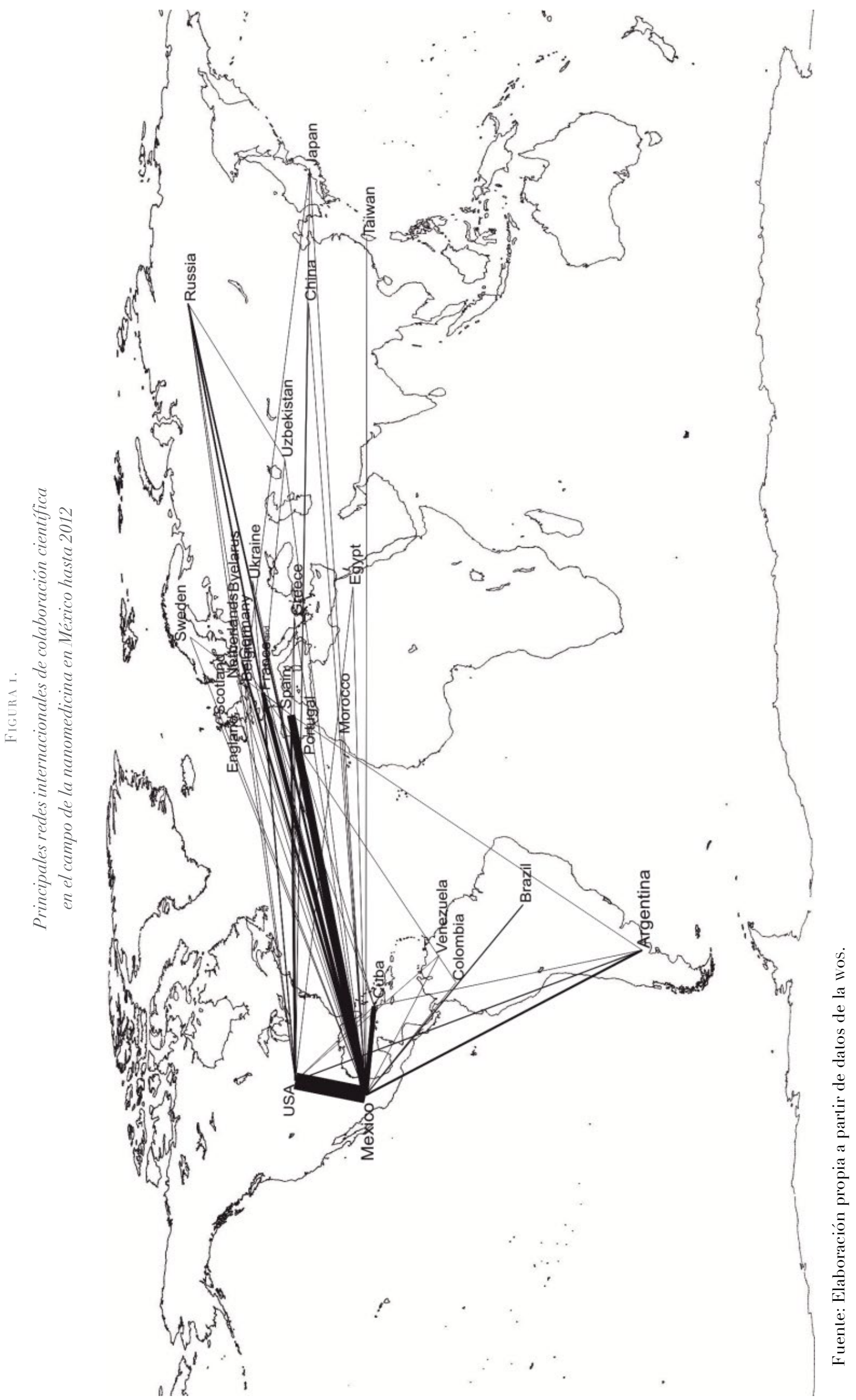


ciones importantes, que de hecho es el tercer país con quien más se ha colaborado en el campo de la nanomedicina.

\section{Conclusiones}

En los resultados expuestos en este estudio hemos constatado que las colaboraciones científicas internacionales en el campo de la nanomedicina en Méxi- co tienen mucho peso. Esto resalta una serie de cuestiones en torno del desarrollo de la nanomedicina en México y sus alcances para cumplir con las promesas que se han anunciado para el desarrollo de estas nuevas tecnologías. Este estudio, finalmente, nos muestra que se requiere una mejor comprensión del desarrollo de las nanomedicinas en México y, eventualmente, medidas para corregir el rumbo y que éstas sirvan para la sociedad mexicana.

\section{- notas $\cdot-$}

' Freitas, R. A. (2005). Current Status of Nanomedicine and Medical Nanorobotics. Journal of Computational and Theoretical Nanoscience, 2, I, pp. I-25.

2. Vidal-Correa, L. y Zayago-Lau, E. (2014). Nanomedicina: expectativas y regulación para el desarrollo. Observatorio del Desarrollo, 2, 8, pp. 3i-35.
${ }^{3}$ Robles-Belmont, e. y Vinck, D. (20II). A Panorama of Nanoscience Developments in Mexico Based on the Comparison and Crossing of Nanoscience Monitoring Methods. Journal of Nanoscience and Nanotechnology, II, 6, pp.5499-55o7.

4 Wagner, V., Hüsing, B., Gaisser, S., Bock, A.-K. (2008). Nanomedicine: Drivers for development and possible impacts. European Science and Technology Observatory.

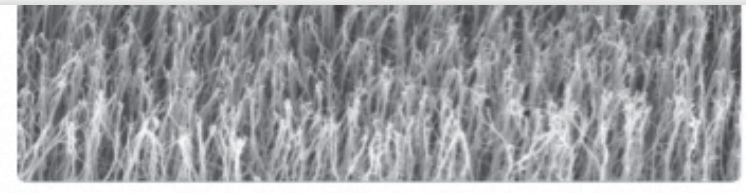

\section{SOCIAL AND ENVIRONMENTAL}

IMPLICATIONS OF NANOTECHNOLOGY DEVELOPMENT IN ASIA-PACIFIC
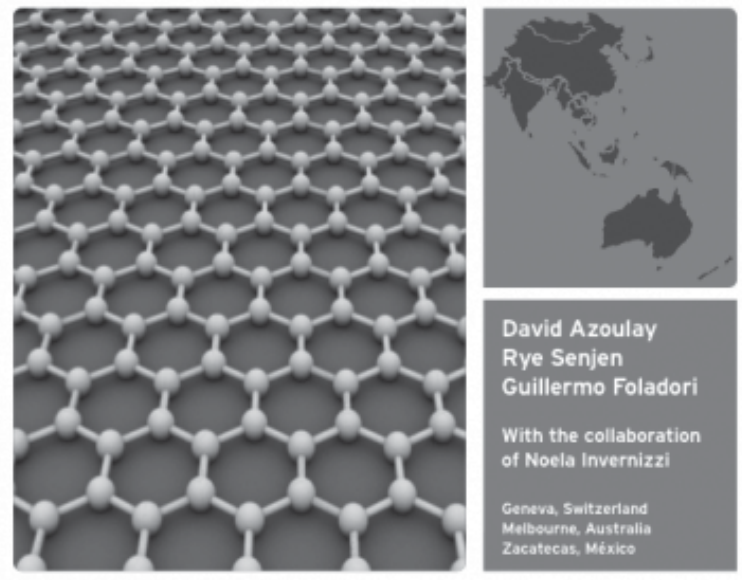

David Azoulay Rye Senjen Guillermo Foladori

With the collaboration of Noela Invernizzi

Ganeva, Sultzerliane

Mentbourne, Austrandi

Zacatecas, México
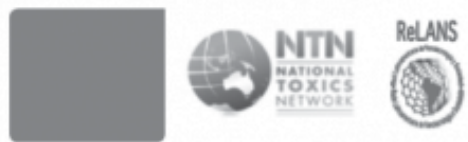

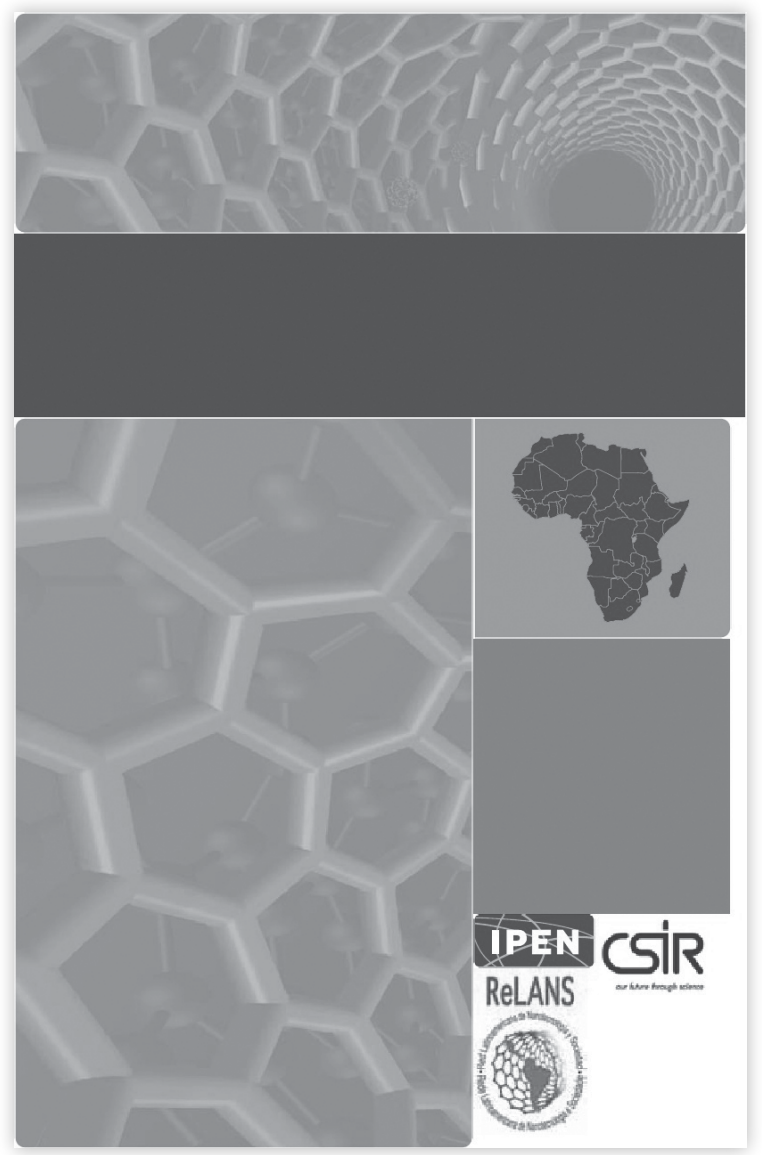


Universidad Autónoma de Zacatecas

Unidad Académica de Estudios del Desarrollo

II Seminario de Universitarios por el Desarrollo La Otra Toma de Zacatecas: Un Siglo Después

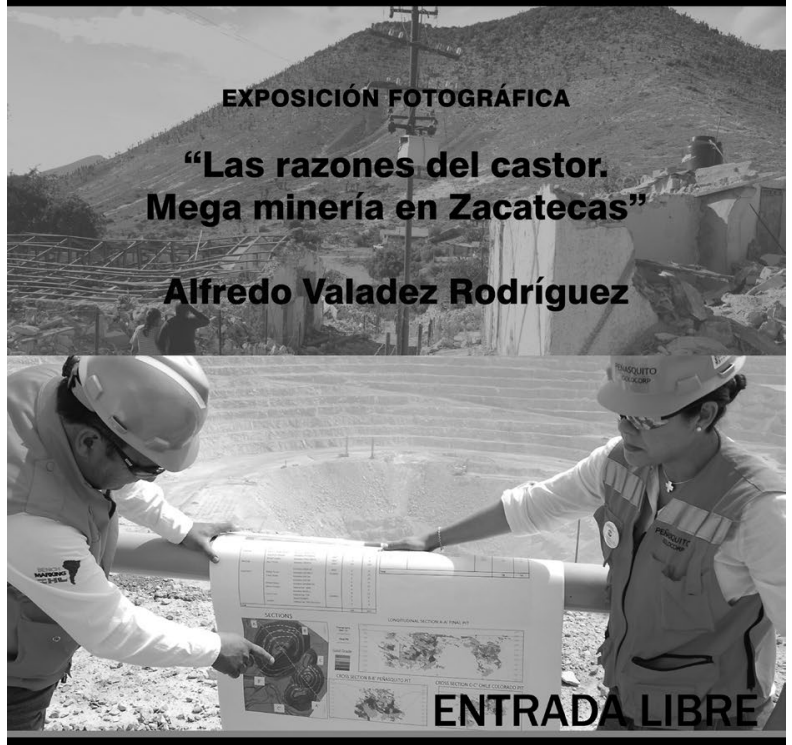

18 - 21 de noviembre de 2014

\section{0:00 - 20:00 hrs.}

Inauguración 18 de noviembre, 20:00 hrs. / Vino de honor

Lugar: Unidad Académica en

Estudios del Desarrollo

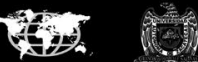

Universidad Autónoma de Zacatecas

Unidad Académica de Estudios del Desarrollo

II Seminario de Universitarios por el Desarrollo

La Otra Toma de Zacatecas: Un Siglo Después

EXPOSICIÓN FOTOGRÁFICA

“Historias intervenidas"

Eduardo Román Quezada

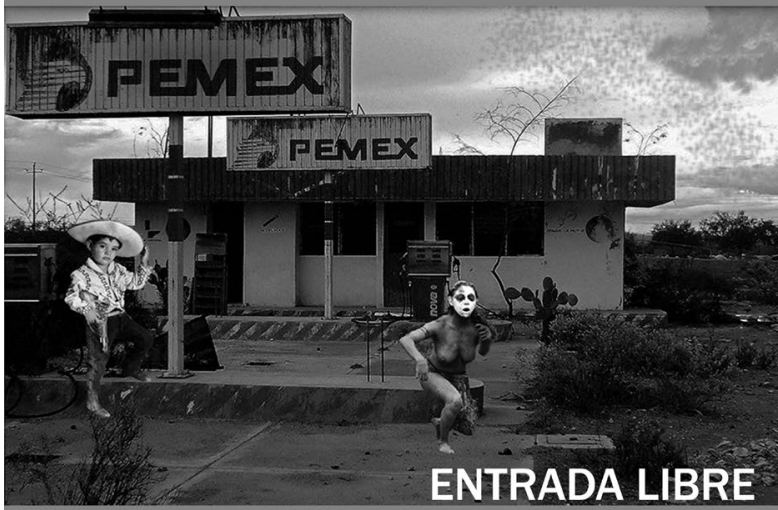

18 - 21 de noviembre de 2014

\section{0:00 - 20:00 hrs.}

nauguración 18 de noviembre, 20:00 hrs. / Vino de honor

Lugar: Unidad Académica en

Estudios del Desarrollo

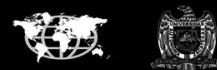

Universidad Autónoma de Zacatecas

Unidad Académica de Estudios del Desarrollo

II Seminario de Universitarios por el Desarrollo La Otra Toma de Zacatecas: Un Siglo Después

EXPOSICIÓN FOTOGRÁFICA

"Ópticas de arte y sociedad, imágenes de nuestro entorno"
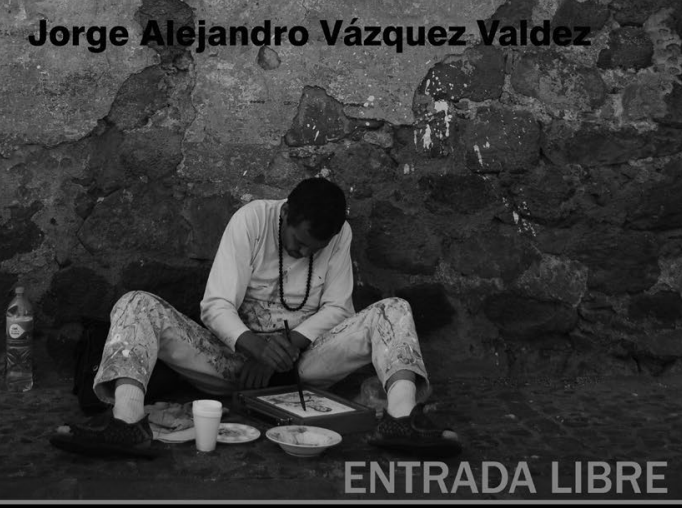

18 - 21 de noviembre de 2014 10:00 - 20:00 hrs.

Inauguración 18 de noviembre, 20:00 hrs. / Vino de honor

Lugar: Unidad Académica en Estudios del Desarrollo

(⿻一丿)
Universidad Autónoma de Zacatecas Unidad Académica de Estudios del Desarrollo

II Seminario de Universitarios por el Desarrollo La Otra Toma de Zacatecas: Un Siglo Después

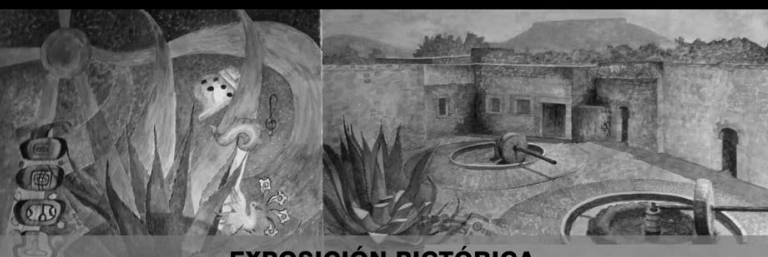

EXPOSICIÓN PICTÓRICA

Imaginarios del mezcal

NICOLÁS MORALES CARRILLO

Docente-investigador del CRUCEN-Universidad Autónoma de Chapingo

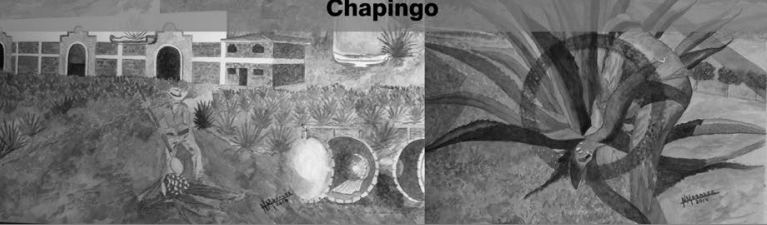

\section{ENTRADA LIBRE}

18 - 21 de noviembre de 2014

\section{0:00 - 20:00 hrs.}

Inauguración 18 de noviembre, 20:00 hrs. / Vino de honor

Lugar: Unidad Académica en Estudios del Desarrollo

सis, 